Rodríguez López, Ramona.

Universitat Politècnica de València.

\title{
Visibilizar, señalar el sonido disruptivo. Soportes para ser escuchados
}

\section{Make visible, point the disruptive sound. Supports to be heard}

TIPO DE TRABAJO: Comunicación.

\section{PALABRAS CLAVE}

Soporte-escucha, altavoces, sonidos disruptivos, smartphones, control usuario, Industria 4.0.

\section{KEY WORDS}

Surface-listening, speakers, disruptive sounds, smartphones, user control, Industry 4.0.

\section{RESUMEN}

En la línea de trabajos que tratan de desarrollar una vía de exploración crítica hacia las interfaces, planteamos la producción de artefactos que hagan preguntas sobre el sonido que acompaña dichas interfaces, sonidos diseñados para mejorar la eficiencia comunicativa en la relación dispositivo-usuario (interacción entre humano-máquina).

Nuestro proyecto consiste en la creación de una serie de piezas en las que se estudia el aspecto sonoro de las interfaces, especialmente las de los smartphones, sacando los altavoces o medios por los que se produce el sonido de su condición de invisibilidad, e indagando en el tipo de sonidos que emiten estos dispositivos, en su mayoría tonos y notificaciones creadas para llamar nuestra atención de manera disruptiva. Estos sonidos forman parte de las estrategias de control de usuario y crean dependencias de las que difícilmente nos podemos sustraer, dada la imposibilidad de cerrar nuestras orejas, y, aunque silenciemos nuestros móviles, siempre estaremos expuestos al sonido de los dispositivos de las personas que estén a nuestro alrededor.

\section{ABSTRACT}

In the line of works that seek to develop a path of critical exploration towards interfaces, we propose the production of artifacts that ask questions about the sound that accompanies these interfaces, sounds designed to improve the communicative efficiency in the device-user relationship (interaction between human-machine).

Our project consists in the creation of a series of pieces in which the sound aspect of the interfaces is studied, especially those of smartphones, taking out the speakers or media through which the sound of their invisibility condition is produced, and inquiring into the type of sounds that these devices emit, mostly tones and notifications created to call our attention in a disruptive way. These sounds are part of the user control strategies and create dependencies that can hardly be subtracted, given the impossibility of closing our ears, and, although we mute our phones, we will always be exposed to the sound of the devices of people who are around us.

\section{INTRODUCCIÓN}

El presente artículo esboza una parte de la investigación realizada en nuestra Tesis Doctoral con motivo de una estancia en el departamento Interface Cultures de la Kunstuniversität Linz. Con una duración de seis meses, tuvimos la oportunidad de presentar el trabajo en las jornadas de carácter semestral PhD-Kolloquium, ofrecidas dentro del PhD-Programm. También presentamos los 
resultados de la investigación en una Guestlecture ${ }^{1}$ organizada por Interface Cultures. En este período, compartimos despacho con artistas, investigadores e investigadoras, estableciendose debates y discusiones en las que se confrontaron opiniones e ideas, enriqueciendo y clarificando nuestra investigación en la tarea de obtener resultados, bajo la modalidad de práctica artística como método de investigación. Cabe reseñar la influencia en el enfoque del tema de investigación del curso You Akademia Project, realizado dentro del programa de emprendimiento de la Fundación Innovación Bankinter en la Ciudad Politécnica de la Innovación, adentrándonos en el mundo de las startups, nuevos planteamientos y modelos de negocio, así como las estrategias de mercado, entre otros conocimientos.

\section{Estado de la cuestión}

El uso de las tecnologías se ha extendido de manera exponencial desde el comienzo del siglo XXI, la velocidad de los cambios se ve acelerada por una sociedad grobalizada que parece haber entrado en una carrera por la innovación, una lógica de progreso infinito que deja poco margen a la reflexión. Vivimos en la que Klaus Schwab denomina cuarta Revolución Industrial o Industria 4.0 que, según el autor, viene marcada por la convergencia de distintas tecnologías como las digitales, las físicas y las biológicas, con un alcance para el que no hay precedentes, afectando a todas las esferas de nuestro mundo influidas por el flujo de información:

Todos los nuevos desarrollos y tecnologías tienen una característica clave en común: aprovechan el poder de penetración que tienen la digitalización y las tecnologías de la información (Schwab, 2016, p. 29).

Hemos pasado de ser productores a ser consumidores, las instituciones panópticas han variado su sistema, frente al comportamiento rutinario y monótono de antaño ahora se impone la lógica del consumo mediante la estrategia de la novedad, exponerlos siempre a nuevas tentaciones manteniéndolos en un estado de ebullición continua (Bauman, Z., 2000, p. 47). En este sentido, Internet y las tecnología digitales están siendo instrumentalizadas para integrarnos en el nuevo orden, sobre todo con el desarrollo de dispositivos con sistemas inteligentes como los smartphones ${ }^{2}$, evolucionando en sofisticacadas y nuevas formas de hibridaciones físico-digitales que crean complejas experiencias, con ecosistemas distribuidos, hiperconectados e inteligentes. Estos dispositivos integran cada vez más dimensiones invisibles con sensores, procesadores, algoritmos, actuadores, materiales inteligentes, etc., que son capaces de tomar nuestros datos e información sin darnos cuenta. Cuentan con interfaces que se sirven de metáforas diseñadas para que resulten imperceptibles en la experiencia de usuario, acompañadas por sonidos de aviso a modo de señales para captar la atención cuando el dispositivo no está a la vista.

Diversos autores están focalizando su análisis hacia las tecnologías digitales desde un punto de vista crítico como por ejemplo Christian Ulrik Andersen y Soren Bro Pold, este último participante en el manifiesto para una aproximación a la critica de la interface (Interface Manifesto) en el que colaboran diversos artistas e investigadores ${ }^{3}$. En relación a lo sonoro hay cada vez más autores que están trabajando sobre el sonido, en los denominados Sound Studies y otras corrientes que se podrían enmarcar dentro del Arte sonoro, o como señala José Iges, piezas artísticas donde el sonido es el elemento principal.

Desde los años 70 Murray Schafer con sus estudios sobre ecología acústica habla del cambio del paisaje sonoro ${ }^{4}$ mundial que introdujo la Revolución industrial y la Revolución eléctrica, suscitando el interés en investigadores de diferentes áreas sobre la relación entre el hombre y los sonidos de su entorno, y qué sucede cuando estos sonidos cambian (Schafer, 2013, p. 19). El teléfono, según Schafer, fue un mecanismo acústico de la Revolución eléctrica que propició muchas transformaciones, además de permitir la comunicación superando la barrera del espacio, el autor lo describe como un dispositivo de gran capacidad para interrumpir el pensamiento (Schafer, 2013, p. 134). En la actualidad los smartphones o máquina de simultaneidades (Colmenares, S. 2019), son los dispositivos más eficaces en cualquier empresa que difusión y captación de información por su alcance 5 .

\section{Hipótesis}

Los avances tecnológicos están cambiando la relación del hombre con los sonidos de su entorno. Las prácticas artísticas se han visto afectadas por el cambio, abriendo una nueva vía de vinculaciones entre la mirada y el sonido capaces de desvelar estrategias sonoras que esconden sistemas de control, entre ellas los sonidos disruptivos.

\footnotetext{
${ }^{1}$ Guestlecture, serie de presentaciones que se realizan por parte de los investigadores invitados en Interface Cultures de la Kunstuniversität Linz, Austria.

${ }^{2}$ Smartphone: palabra inglesa para designar a los teléfonos inteligentes

${ }^{3}$ Rescarado el 9 de febrero de 2019, de: https://interfacemanifesto.hangar.org/index.php/Main Page/es

${ }^{4}$ Paisaje sonoro: término que acuñado por Murray Schafer en el libro El paisaje sonoro y la afinación del mundo. El autor lo concibe como un ecosistema integrado por todos los acontecimientos acústicos del mundo, el catálogo de los ruidos y sonidos entre los cuales vivimos.

${ }^{5}$ La estadística de número de usuarios de teléfonos inteligentes a nivel mundial es de 2659,4 millones, aunque el número de tarjetas SIM supera la población mundial (más de 5000 millones). Estas cifras nos da una idea de la importancia de estos dispositivos. Rescatado el 8 de febrero de 2019, de: https://es.statista.com/estadisticas/636569/usuarios-de-telefonos-inteligentes-a-nivel-mundial--2019/
} 


\section{Objetivos}

Poner en foco en la escucha, a través de la experimentación y la construcción de artefactos o piezas sonoras, utilizando elementos de los smartphones como los altavoces o haciendo referencia a los mensajes que nos llegan de estos dispositivos.

Analizar el papel del sonido en las tecnologías digitales de los teléfonos inteligentes y sus interfaces.

Reflexionar sobre los nuevos sonidos disruptivos creados por las tecnología móviles, como una manera de concienciar-nos, o promover una actitud crítica hacia estas herramientas o aplicaciones que estamos incorporando en nuestras vidas.

\section{METODOLOGÍA}

La metodología planteada se basa en el estudio teórico-técnico necesario para la creación de artefactos y propuestas visuales, reutilizando componentes y elementos gráficos propios de los móviles y sus sonidos, como diría José Iges, organizar con intención artística el sonido en el tiempo y el espacio (Iges, 2017, p. 19) para cambiar su sentido. Son referentes importantes, además de Murray Schafer, los trabajos de Brandon Labelle, tanto sus proyectos que vincula prácticas artísticas sociales y arte sonoro, como sus libros y escritos en los que centra su atención en la forma en que el sonido circula a través del entorno construido, condiciona los espacios arquitectónicos y constituye la base de los proyectos sociales y culturales (Labelle, 2010). Otra fuente referencial son los Sound Studies cuyo objeto de estudio es el entorno sonoro de una manera multidisciplinar y desde varios campos de estudio, ligado a una práctica tecnológica en paralelo a la artística, explorando más asiduamente la relación entre la escucha y la mirada (Iges, p. 20). Algunas webs destacadas de consulta son: The Journal of Sonic Studies ${ }^{6}$ y Sound Studies $l a b^{7}$.

En las cuestiones técnicas del proyecto, además de los talleres presenciales realizados durante la estancia en Linz, hemos visitado distintos portales accesibles en internet con tutoriales e indicaciones sobre electrónica y computación, otra fuente de información la hemos obtenido de los cursos online (tanto gratuitos como de pago) de Edx, Udemy y Kadenze.

Hay un gran número de artistas trabajado con presupuestos similares a nuestro proyecto de investigación. En el presente artículo citaremos los que se acercan más a las propuestas que más adelante detallaremos, bien por el uso de los altavoces como elementos formales de las instalaciones o esculturas sonoras, o por haber tratado la temática de los smartphones de forma directa o indirecta. Un referente importante es Golan Levin y Colaboradores con piezas como Dialtones (A Telesymphony) presentado en Ars Electrónica 2001, en las que se explora el potencial musical de este ubicuo dispositivo ${ }^{8}$. Otra obra más centrada en los mensajes es Spam Tower (2012) de Ricardo Iglesias, una torre compuesta de móviles por la que emite mensajes publicitarios y SMS, haciendo alusión a las nuevas formas panópticas mecánico-electrónicas en las que el control es ejercido por conglomerados económicamente gobalizados, donde el concepto de individuo es reducido a sus datos virtu-reales ${ }^{9}$. Rafael Lozano-Hemmer tiene varios proyectos en los que ha empleado altavoces con distintas propuestas formales, en la instalación Pan-Anthem (2014) coloca en una pared una serie de altavoces organizados para visualizar una estadística de datos, el sonido que se escucha es el de cientos de imnos nacionales de países que se accionan con la proximidad del espectador, no trata de una estadística en particular, sino del potencial de visualizarlas de forma sonora (Labastida, A. 2015, p. 17). Otra obra es Sphere Packing, una serie de esferas compuestas por un número concreto de auriculares por las que suena la música de distintos autores. El espectador se tiene que acercar para poder escuchar la música, con la distancia lo que se escucha es una especie de murmullo. ${ }^{10}$

\section{DESARROLLO}

Para acercarnos al sonido de las interfaces de una manera crítica se han seguido directrices empleadas por referentes del ámbito sonoro y visual, sobre todo las estrategias propuestas en Interface Manifesto ${ }^{11}$. En las piezas se desarrollan dos apartados: por una parte la cuestión material o forma del objeto y, por otra, los sonidos que se van a escuchar por los artefactos o soportes de escucha, estableciendo asimismo dos categorías: lo Aural en donde se situaría el trabajo con distintos tipos de sonidos, y lo Visual, en el que se despliegan los materiales que van a servir de soporte y medios de reproducción, en este caso altavoces de móviles.

\footnotetext{
${ }^{6}$ Revista de estudios sonoros. https://www.researchcatalogue.net

${ }^{7}$ http://www.soundstudieslab.org/

${ }^{8}$ Dialtones (A Telesymphony). 28 minutos de concierto producido por los tonos de llamada de 200 visitantes en tiempo real. Extracto y descripción traducido del informe final del proyecto. Rescatado el 18 de febrero de 2019, de: http://www.flong.com/storage/pdf/reports/dialtones report.pdf

${ }^{9}$ Información extraída de la web del artista. Rescatado el 25 de febrero de 2019, de: http://www.ricardoiglesias.net/2012/03/spam-tower/

${ }^{10}$ Rescatado el 15 de febrero de 2019, de: http://www.lozano-hemmer.com/

${ }^{11}$ Rescatado el 15 de febrero de 2019, de:

https://interfacemanifesto.hangar.org/index.php/Main_Page/es\#Propuesta_de_acciones_relacionadas_con_los_puntos_del_manifesto
} 
La inspiración para seleccionar el material de soporte es la cámara anecoica visitada por John Cage en 1951 esperando escuchar el silencio (estos recintos cerrados están aislados del exterior con espumas porosas). Además de la capacidad de esta espuma acústica fonoabsorvente para evocar lo sonoro por su uso, tiene una geometría ondulada que recuerda a las ondas sonoras. En cuanto al empleo de los altavoces de los móviles, se trata de hacer evidente el medio sonoro de estos dispositivos relegados al estatus de invisibilidad, sacándolos de su rol subsidiario se pretende activar la capacidad de suscitar preguntas, en primera instancia, al no ser reconocidos como altavoces por no tener la forma que todos recordamos de estos medios, se establece la pregunta ¿qué es esto? ${ }^{12}$

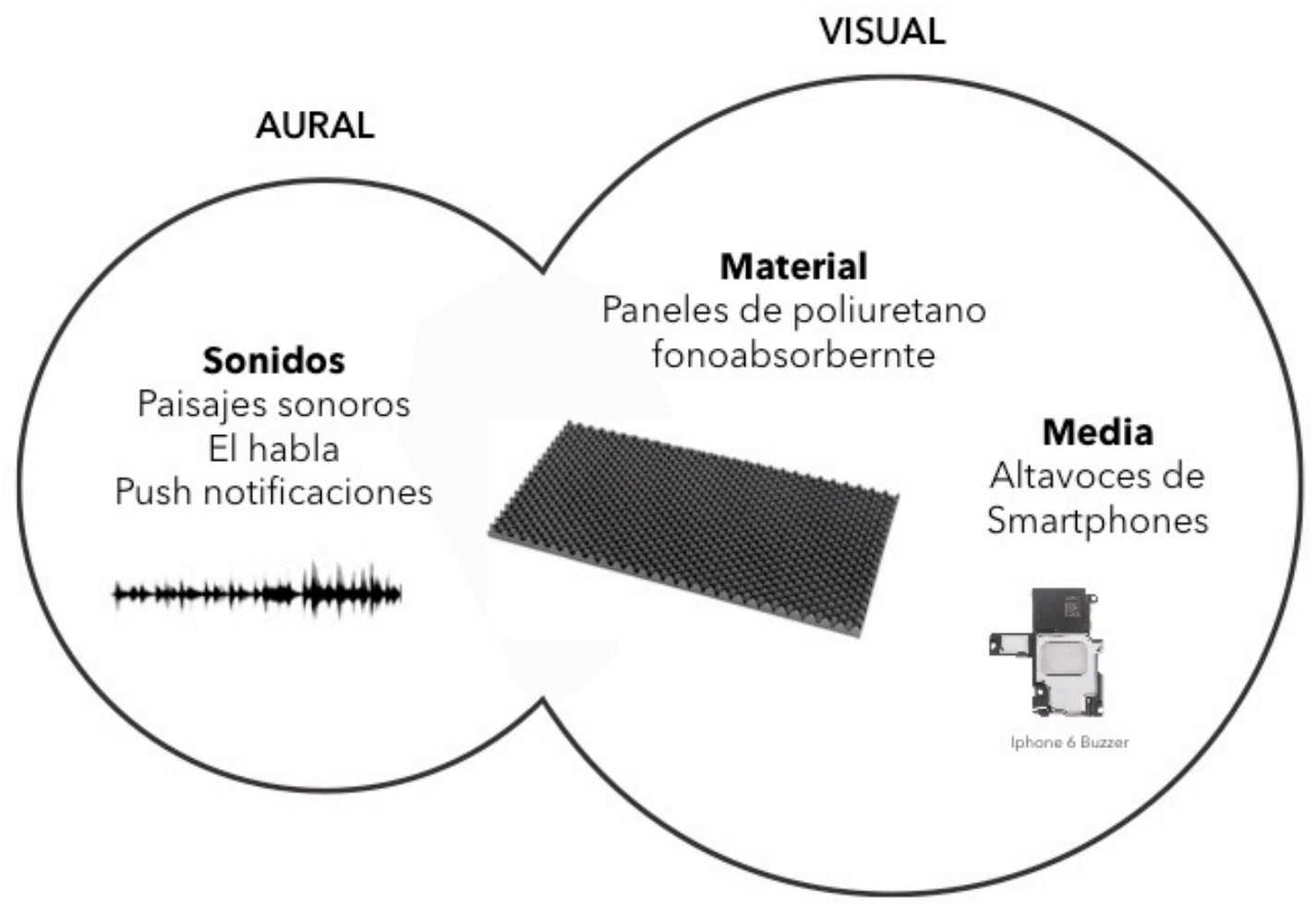

Figura 1. Esquema de los elementos de estudio

\section{Mobile Speaker Panel \#6_30}

Pieza compuesta por un soporte rectangular de $100 \times 40$ centímetros en el que se han insertado 6 grupos de altavoces de móviles de 30 modelos diferentes. Dispone de un sensor fotosensible que proporciona datos lumínicos. El sonido está gestionado desde la placa NodeMCU V2 ESP8266 con el reproductor de audio Dfplayer Mini, estos se conectan con los altavoces ofreciendo 6 salidas de audio independientes. La placa es programada con el IDE de Arduino obteniendo los datos del DFplayer que reproduce un archivo de sonido y del sensor que gestiona el volumen. El resultado es un panel con 6 sonidos a un volumen bajo que se ve aumentado cuando acercamos nuestro oído al sensor que está en medio de los altavoces. La idea es ofrecer una experiencia de escucha de cercanía, en la línea de Sphere Packing de Lozano-Hemmer. Los 6 audios se perciben como murmullos y se hace necesario acercarnos para escuchar el mensaje que de cada canal sonoro.

\footnotetext{
${ }^{12}$ Hemos llegado a esta conclusión después de mostrar los altavoces a distintas personas sin estar emitiendo sonido. Cuando se ha mostrado en funcionamiento en un porcentaje alto también ha habido una extrañeza hacia los altavoces sin tener claro que el sonido procediera de estos dispositivos.
} 


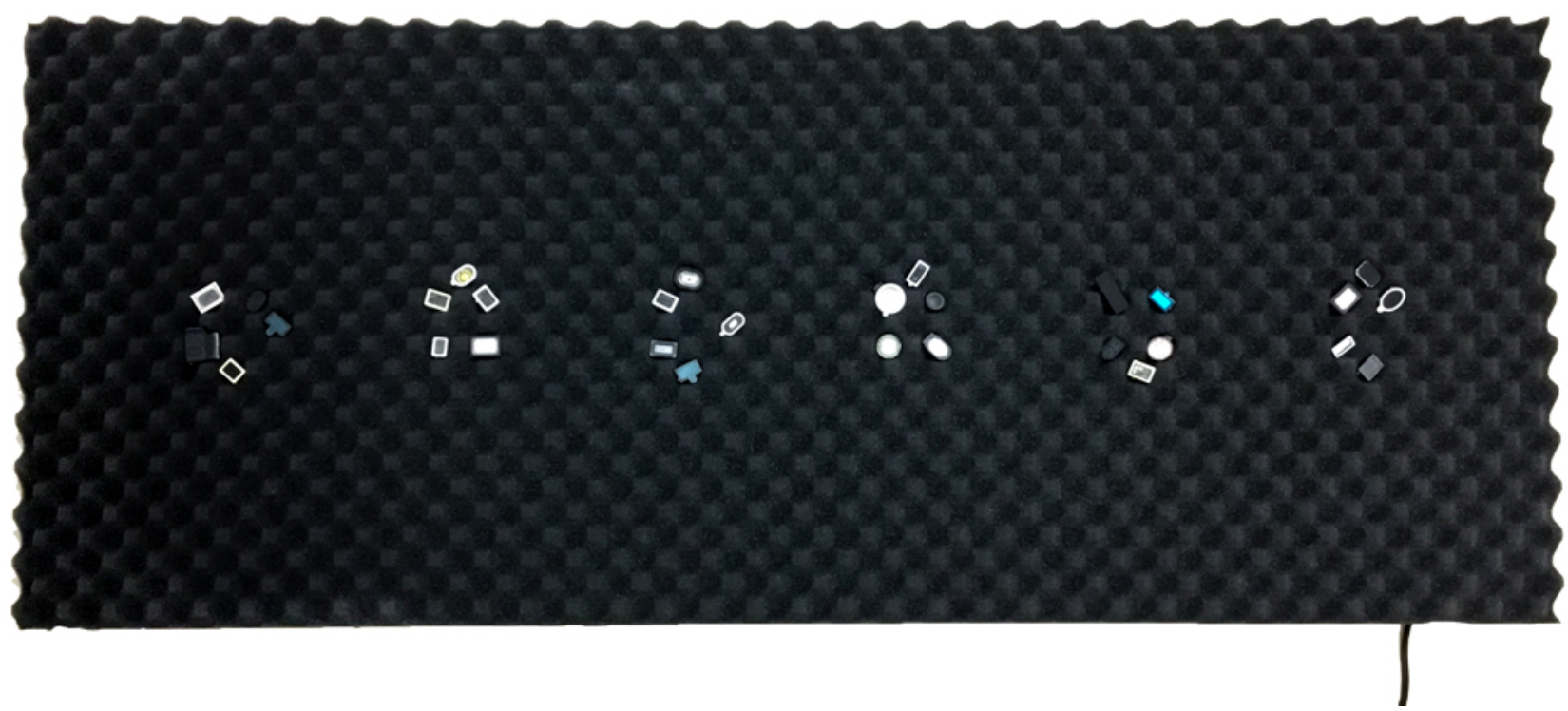

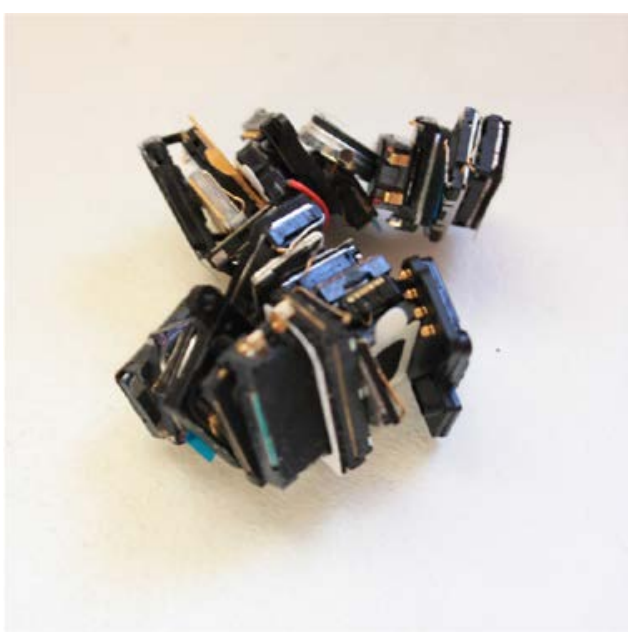

Figura 3. Imagen de los altavoces

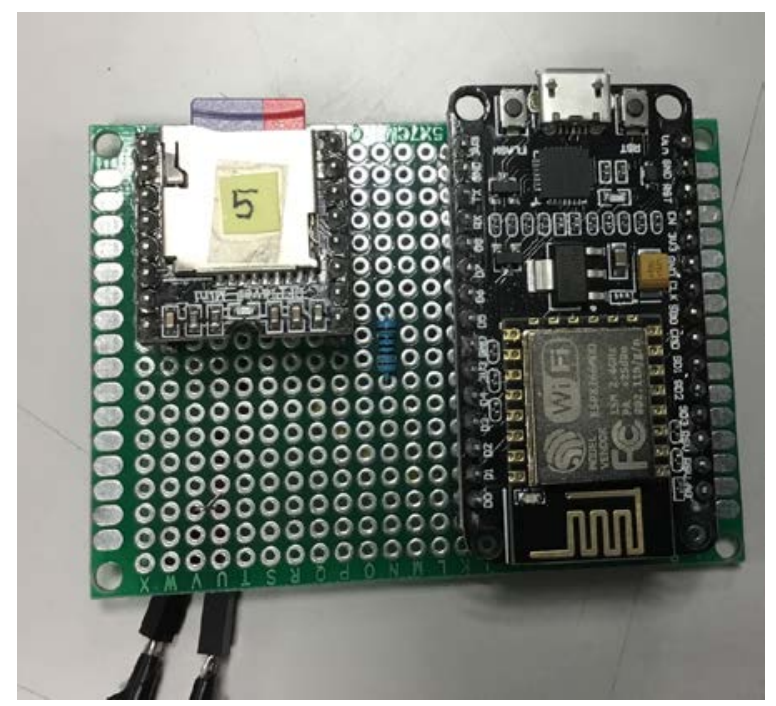

Figura 4. Detalle del dispositivo: NodeMCU v2 ESP8266, Dfplayer Mini, resistencia, tarjeta mini SD

En el apartado Aural ${ }^{13}$ hemos trabajado en seis propuestas para reflexionar sobre la escucha: a través de la creación de un paisaje sonoro global compuesto de sonidos de la ciudad ${ }^{14}$, de las notificaciones de los móviles, incorporando su función de señales de alerta y cualidades disruptivas, y de la voz, explorando la tonalidad de las aplicaciones de internet.

\footnotetext{
${ }^{13}$ El apartado Aural tiene un carácter de especificidad, pudiendo ser modificado en función de la variación del contexto expositivo. En la propuesta Mobile Speaker Panel \#6_30 el contexto sonoro responde a la residencia en la ciudad de Linz.

14 Uso de grabaciones de la ciudad de Linz consonancia con los proyectos de Brandon Labelle en los que establece relaciones entre sonido y espacios sociales.
} 


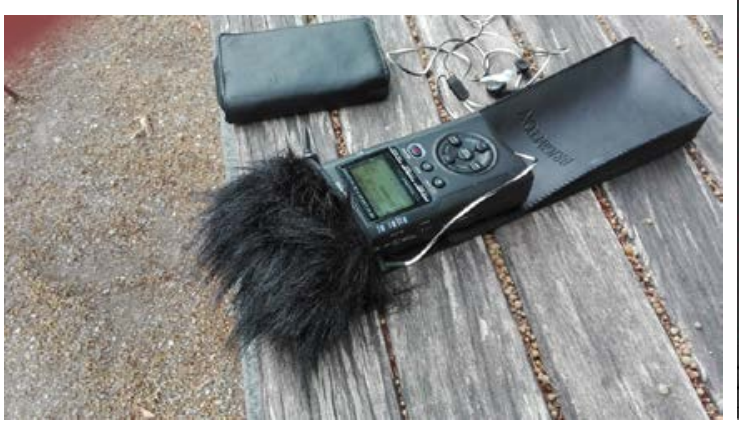

Figura 5. Imagen durante los paseos sonoros.

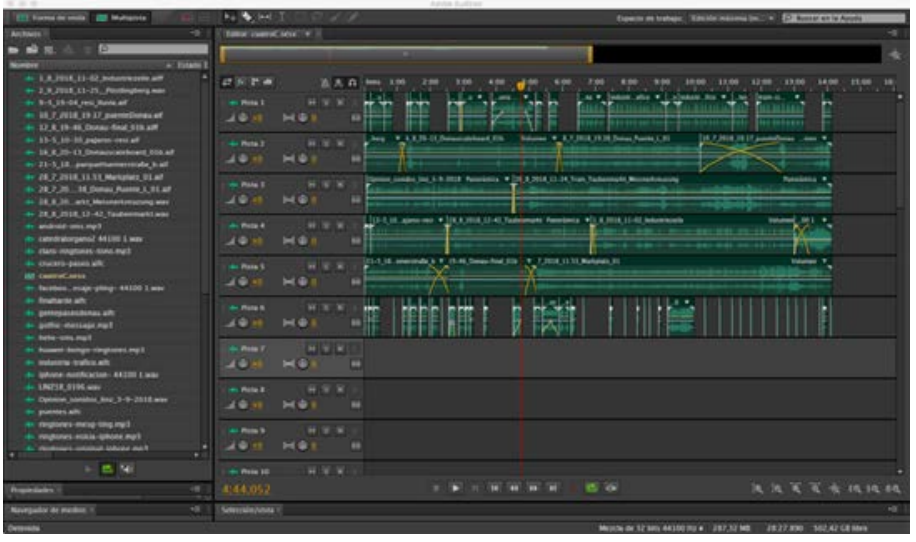

Figura 6. Captura de pantalla, edición multipista en Adobe Audition.

Sonidos:

- Uno, dos y tres. Proceden de grabaciones de la ciudad de Linz realizadas durante la estancia de investigación. Selección de sonidos singulares que reflejan el ritmo percibido de la ciudad y sus habitantes, sus lugares, parques, río, tranvía, mercado, entre otros. Componen fragmentos de realidad que descontextualizada y remezclada buscan la evocación de sensaciones y recuerdos.

- Cuatro. Catálogo de sonidos escuchados después de realizar un ejercicio de limpieza de oídos, afinando la escucha y detectando los distintos sonidos de cada grabación. Listado de palabras convertidas en sonido mediante la aplicación de Traductor de Google. Con esta propuesta indagamos en la voz de la máquina y su humanización (cada vez más servicios de voz como Alexa o Siri), explorando aspectos como el tono, la amplitud y el timbre.

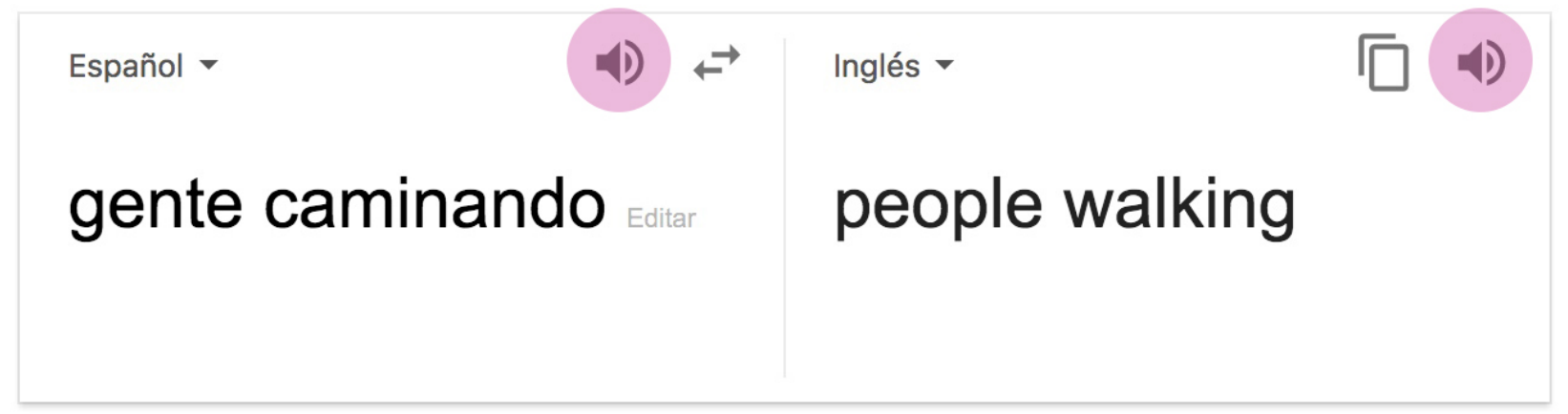

Abrir en el Traductor de Google

Enviar comentarios

Figura 7. Captura de pantalla de la aplicación Traductor de Google.

- Cinco. Aprovechando la visita de un grupo de amistades que visitaron la ciudad de Linz durante unos días, realizamos una entrevista preguntándoles por los sonidos de la ciudad. En este caso, el objetivo era resaltar la escucha del turista y la percepción general del sonido del lugar (durante la visita se plantearon temas sobre el paisaje sonoro y escucha).

- Seis. Selección de sonidos de notificaciones y alertas de distintos modelos de móviles descargados de internet, mediante el uso de los sonidos de notificación queremos que el usuario interrumpa su pensamiento transportándolo a los momentos asociados a dichos sonidos ${ }^{15}$.

\section{Panel \#12_iphone6}

Panel con un soporte de $70 \times 70$ centímetros y 12 grupos de 4 altavoves del iphone 6 . Cada grupo reproduce un sonido que es gestionado por una placa y su reproductor DFplayer Mini y un sensor de movimiento.

\footnotetext{
${ }^{15}$ Como la obra de Lozano Hemmer Pan-himno, que utiliza la referencialidad del sonido de los imnos para conectar ideas.
} 
Proyecto con 12 canales independientes de sonido, reproduciendo el tono genérico de las notificaciones del iphone 6 . Hay un trabajo de deconstrucción de la melodía que es dividida en partes, va sonando por cada canal de manera aleatoria con un volumen aleatorio también. El pasa a ser un elemento sonoro en el que no se reconoce su melodía dislocando su función. El soporte se convierte en una especie de instrumento de ruido que se acciona con la presencia del espectador.

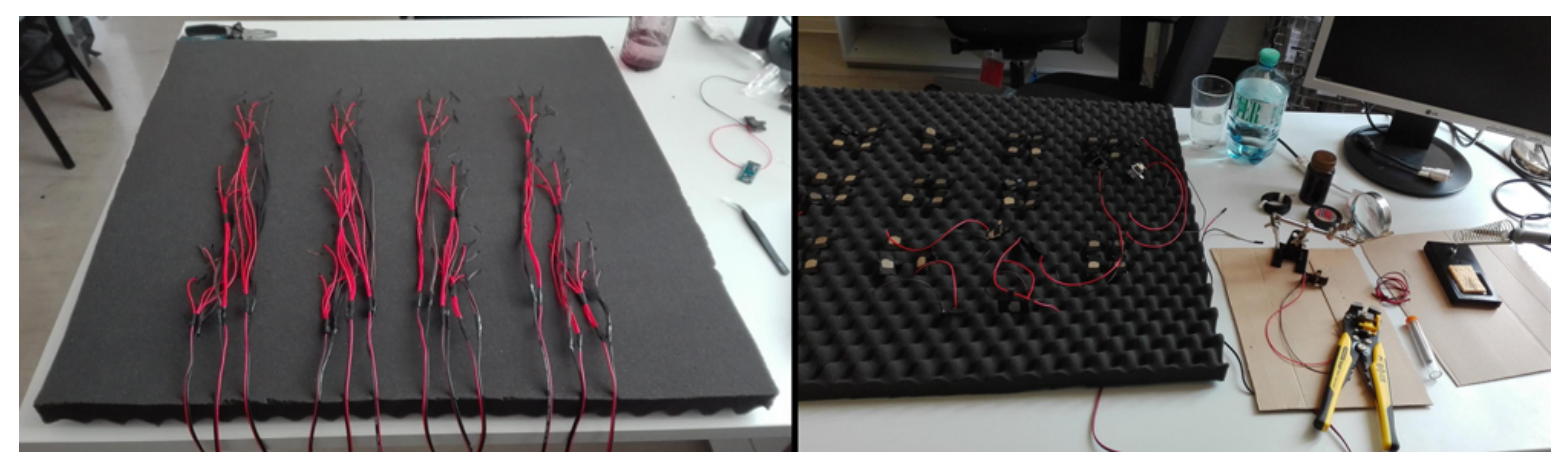

Figura 8. Imágenes del proceso de trabajo

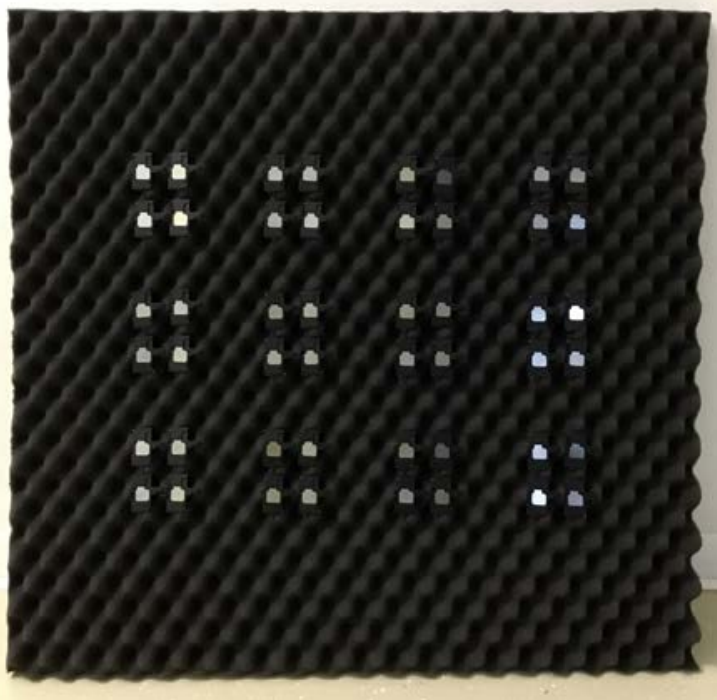

Figura 9. Panel \#12_iphone6

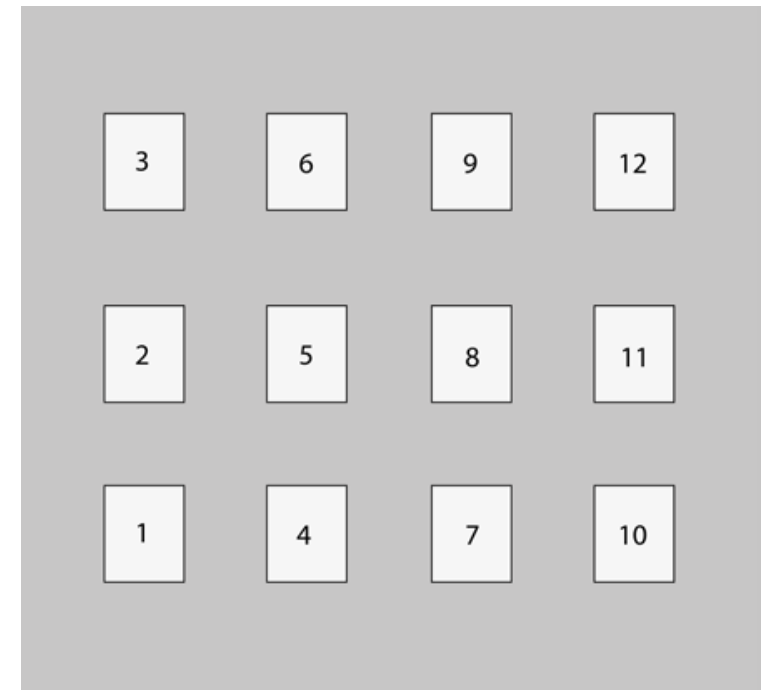

Figura 10. Esquema de canales sonoros del Panel \#12_iphone6

\section{CONCLUSIONES}

Después de la investigación expresada en el presente artículo hemos podido extraer algunas conclusiones que pueden servir a modo de respuesta a nuestra hipótesis. En primer lugar las nuevas tecnologías relacionadas con los dispositivos móviles y teléfonos inteligentes están cambiando nuestro entorno sonoro, Murray Schafer y diferentes investigadores han ido analizando los cambios que en la actualidad desde los Sound Studies y el Arte sonoro se abordan desde distintas áreas. Son importantes también las aportaciones de Interface Manifesto, en cuanto que generan un contexto crítico de publicaciones y trabajos artísticos que son fuente referencial y conectan con nuestra idea de crear soportes para la reflexión.

En relación a las piezas anteriormente descritas Mobile Speaker Panel \#6_30 y Panel \#12_iphone6, tuvimos acceso a discusiones con otros artistas e investigadores durante la presentación de nuestro trabajo en Interface Cultures. El objetivo era que los oyentes reflexionaran sobre los sonidos que escuchaban de sus dispositivos móviles y el grado de interferencia en su día a día, como resultado recibimos muestras de gran interés hacia el tema y los proyectos mostrados (se consiguieron contactos para compartir la evolución del trabajo). 
La investigación cuenta con otras líneas en fase de desarrollo como son: un wearable o vestido confeccionado con esponja acústica y altavoces anexados, esta pieza está pensada para la realización de una performance y un recorrido, en un acto de invasión e incursión en distintos espacios. Otra propuesta en la que estamos trabajando es la realización de un taller teórico-práctico para promover el acercamiento hacia la reflexión sobre los sonidos disruptivos, planteando entre sus objetivos principales la creación de una pieza colectiva para ser exhibida.

\section{FUENTES REFERENCIALES}

Bauman, Z. (2000). Trabajo, consumismo y nuevos pobres. Barcelona: Gedisa, D.L.

Colmenares, S. (2019). Apuntes para una Psicología Social del Smartphone. Rescatado el 15 de febrero de 2019 , de: https://ssociologos.com/2019/02/06/apuntes-para-una-psicologia-social-del-smartphone/

Iges, J. (2017). Conferencias sobre arte sonoro. Madrid: Ardora.

Labastida, A. (2015). Tercero incluido o La máquina y su doble. Rafael Lozano-Hemmer: Pseudomatismos/Pseudomatisms (pp. 13-20). Catálogo. MUAC, Museo Universitario Arte Contemporaneo. Recuperado el 15 de febrero de 2019, de: http://www.lozanohemmer.com/texts/bibliography/monographs/catalogue pseudomatisms.pdf

Lozano-Hemmer, R. (2013). Sphere Packing. (2013). Recuperado el 15 de febrero de 2019, de: http://www.lozanohemmer.com/sphere packing.php

Labelle, B. (2010). Acoustic Territories: Sound Culture and Everyday Life. Continuum Books.

Schafer, R. Murray. (2013). El paisaje sonoro y la afinación del mundo. Barcelona: Intermedio.

Schwab, K. y Botín, A. P. (2016). La cuarta revolución industrial. Barcelona: Penguin Random House, D.L. 